\title{
Evaluation of the Aircraft Distribution in Satellite Spotbeams
}

\author{
Christoph Petersen, Maciej Mühleisen, and Andreas Timm-Giel \\ Institute of Communication Networks \\ Schwarzenbergstr. 95, 21073 Hamburg, Germany \\ $\{\mathrm{chr}$.petersen, maciej.muehleisen, timm-giel\}@tuhh.de \\ http://www.tuhh.de/comnets
}

\begin{abstract}
Upcoming inflight entertainment and other onboard data services request broadband data connections during long- and short-haul flights all over the world. To cope with this amount of traffic, especially over oceanic areas where no ground infrastructure is available, satellite systems offer broadband satellite links for avionic purpose.

This work analyzes the distribution of aircraft traffic for selected satellite spotbeams for intercontinental, rural and urban scenarios. For this, the statistic properties of the number of arriving and departing aircraft within a time interval are analyzed. Our results show, that the law of small number and therefore a Poisson distribution is only applicable to areas with low air traffic. Aircraft arrivals to- and departures from satellite spotbeams can be modeled using the negative binomial distribution, as proven by presented results.
\end{abstract}

\section{Introduction}

Aircraft are fast and popular conveyances today. For safety and passenger comfort, broadband data communication links are often installed. In some regions, but only over terrain, direct air to ground links are maintained, e.g. Aircell GoGo in the United States of America. Other avionic data links as VHF or HF do not offer high bandwidth, are highly congested and very expensive, so they are not used for services requesting high data rates. Therefore, the most widely used worldwide available avionic broadband data communication are satellite links. Fields of application range from inflight entertainment to maintenance or even flight recorder data transmission. To assess the performance of current satellite systems and estimate the capacity demands, reliable information about the number of aircraft inside specific areas are required.

In order to estimate the aggregated capacity demands, the actual offered data traffic load needs to be analyzed. It is feasible to assume the offered data traffic load being proportional to the number of aircraft in that area. Although traces of aircraft densities are available, analytical models allow more general conclusions about offered traffic loads and help to become independent from large data sets of aircraft positions. Also conclusions about areas where no aircraft position information are available may be drawn. These models are also suitable to

T. Bauschert (Ed.): EUNICE 2013, LNCS 8115, pp. 46-53, 2013.

(C) IFIP International Federation for Information Processing 2013 
extrapolate prospective situations or determine system performance for extraordinary situations, e.g. aviation distress. Furthermore, an analytical model can be used for systems simulation.

The same methods were applied to derive realistic vehicular traffic models. Examplarily in [1, a model for car arrivals on higways around the city of Madrid, Spain, is developed. The analysis of the arrival process identifies two different types of vehicles to be further investigated: Cars that drive together in a bursty way and isolated ones without any dependencies e.g. in traveling speed. Therefore, a Gaussian-exponential mixture model is proposed to handle bursty traffic with Gaussian distributed interarrival times and isolated traffic with exponential distributed interarrival times. For sparse vehicular traffic, 22 comes to the same result of exponential distributed vehicular interarrival times and therefore Poisson distributed vehicle arrival rates.

In 3 the German Aerospace Center (Deutsches Zentrum fuer Luft- und Raumfahrt - DLR) developed an aircraft distribution model to evaluate aircraftto-aircraft communication. In [4] the feasibility of that approach is shown, based on data from the airline flight schedule database published by the International Air Transport Association (IATA) and therefore, assuming all aircraft being equipped with ad-hoc communication equipment. The analysis presented in the following is based on more realistic aircraft position data derived from Flightradar24 [5].

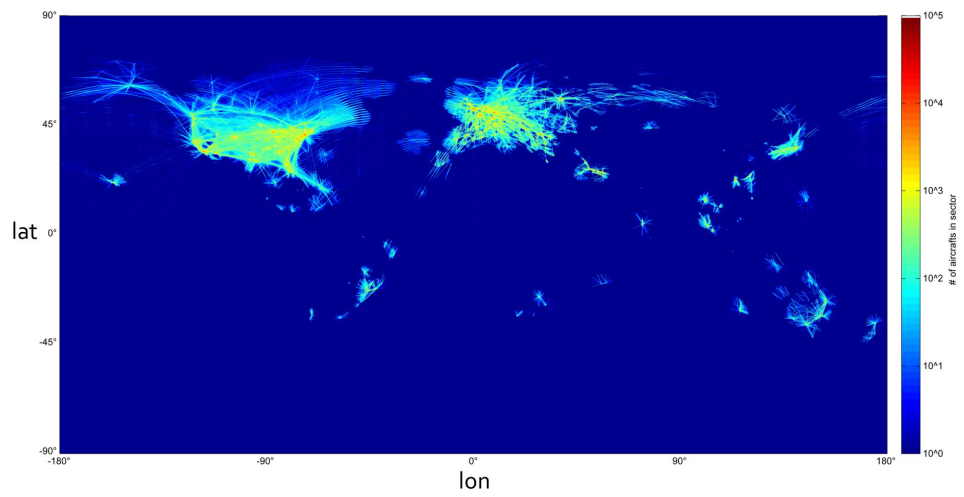

Fig. 1. Two weeks of captured Flightradar24 data

\section{Captured Data Set and scope}

Flightradar24 captures and visualizes Automatic Dependant Surveillance Broadcast (ADS-B) messages from aircrafts, received by the Federal Aviation Administration (FAA) and a community of people privately operating ADS-B receivers. The available aircraft position data does not cover the whole globe. In fact, oceanic areas are very rarely covered as visible in Figure 1, showing all observed 
aircraft position during a two week period. Conclusions regarding aircraft densities over the ocean can be derived from aircraft position data close to the ocean shore. This is visible in Figure 2, representing the observed aircrafts with scope over the United States of America.

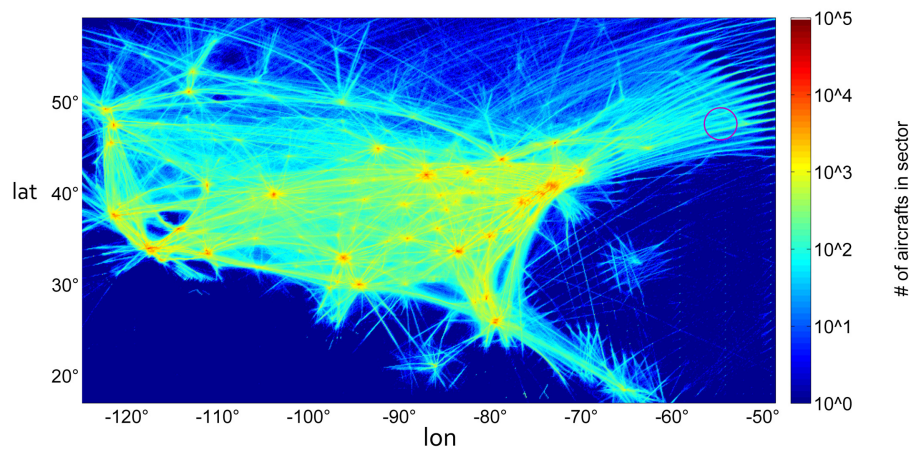

Fig. 2. Spotbeam over Newfoundland

\section{Aircraft Arrival Process}

In order to derive worst case capacity demands, the busy hour of each day is analyzed. The area of interest for this model is a satellite spotbeam with a satellite system specific radius. Considering one particular day, the quadruple $d=\{l$ lat,lon, $t, i d\}$ represents one data record with the unique aircraft ID, the aircraft position in geographic coordinates lat, lon at time instance $t$. The set $\mathbb{D}_{t}$ contains all data records worldwide at time $t$, whereas the subset $\mathbb{D}_{t, A} \subset \mathbb{D}$ is reduced to the records inside the satellite spotbeam area $A$, defined by a radius $r$ around its center point lat , lon $_{C}$. Furthermore, the subset $\mathbb{D}_{t, A, H} \subset \mathbb{D}_{t, A}$ describes all data records of $\mathbb{D}_{t, A}$ within the given busy hour of an arbitrary day in this area. To identify the aircraft arrivals and departures, the set $\mathbb{X}_{t, A, H}$ is created containing all aircraft ID records from $\mathbb{D}_{t, A, H}$ of time instance $t$. The update interval of the captured flight data is limited to the update interval of Flightradar24 being $\Delta t=10 \mathrm{~s}$. Therefore, a time-discrete model describing the aircraft arrivals and departures during one time instance $\Delta t$ is developed. With $\mathbb{X}_{t, A, H}$ and $\mathbb{X}_{t+\Delta t, A, H}$ as the set of aircraft for the next time instance,

$$
a(t)=\left|\mathbb{X}_{t+\Delta t, A, H} \backslash \mathbb{X}_{t, A, H}\right|
$$

is the number of aircrafts (IDs) being in that area at time instance $t+\Delta t$ but not at time instance $t$. Hence, $a(t)$ is the number of aircraft arrivals in area $A$ during that update interval. Analogously, the number of departures from the area is defined as

$$
d(t)=\left|\mathbb{X}_{t, A, H} \backslash \mathbb{X}_{t+\Delta t, A, H}\right|
$$


The data for $a$ and $d$ is collected and analyzed for several weeks. Figure 3 shows the distribution of aircraft arrivals inside a $200 \mathrm{~km}$ radius of Newfoundland. It allows the hypothesis that the number of aircraft arrivals follow a Poisson distribution. This hypothesis is verified using the Chi Square test.

\section{Scenarios}

Several scenarios for the Iridium [6], [7] low earth orbit satellite systems are further investigated. The aircraft arrival process for one spotbeam is analyzed. Depending on its position inside the overall satellite footprint, the area of the spotbeam equates to a circle becoming oval towards the border of the footprint. In this work, a circle with radius $r$ and according area $A$ is assumed. The system specific radius is $r_{\text {Iridium }}=200 \mathrm{~km}$ for the Iridium system.

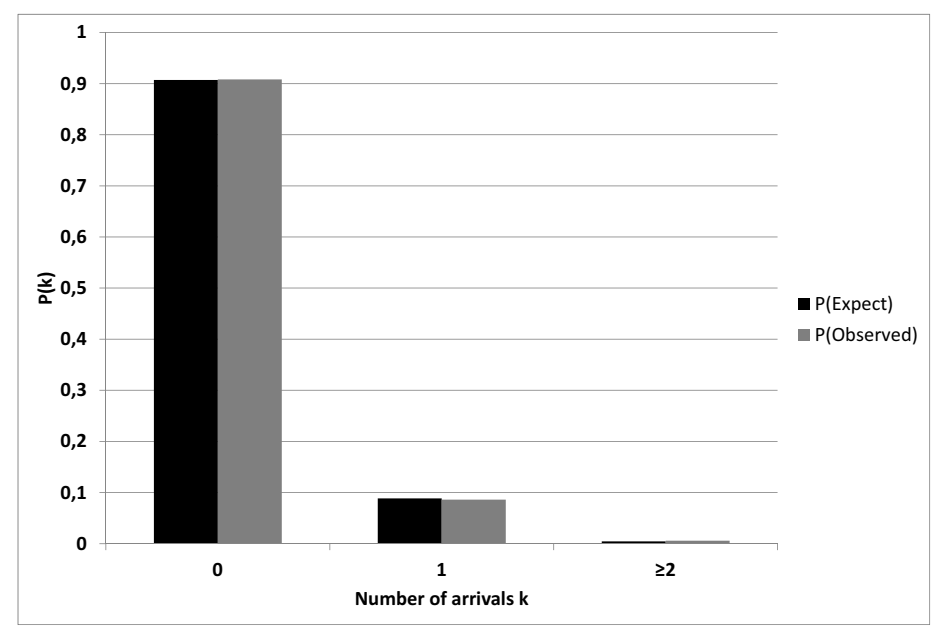

Fig. 3. Observed arrivals over Newfoundland against Poisson hypothesis, $r=200 \mathrm{~km}$

The parameters for the model differ depending on the flight scenario and e.g. the used communication system. As flight scenarios, data for urban, rural or intercontinental areas is analyzed. The transition beetween these scenarios is smooth, but as typical representatives a spotbeam over New York City has been chosen for the urban scenario, over Omaha in Nebraska for the rural scenario and over the ocean shore of Newfoundland as intercontinental scenario.

\section{$5 \quad$ Results}

Figure 3 shows the distribution of aircraft arrivals over Newfoundland for the intercontinental scenario as well as the theoretical distribution according to a 
Poisson distribution. For Chi Square testing, $k$ classes are defined and the class number, except for the last class $k$, represents the number of arrivals during a time instance. The empirical rate parameter $\hat{\lambda}$ is set to the average number of arrivals within the considered timeframe and busy hours. The $k^{\text {th }}$ class summarizes the occurences of even more or equal than $k-1$ arrivals to avoid classes with to few arrivals.

As visible in the figure, only a few aircraft pass this area. The result is based on two weeks of flight data in December 2012. The Chi Square test supports the hypothesis of aircraft arrivals being Poisson distributed with $95 \%$ confidence.

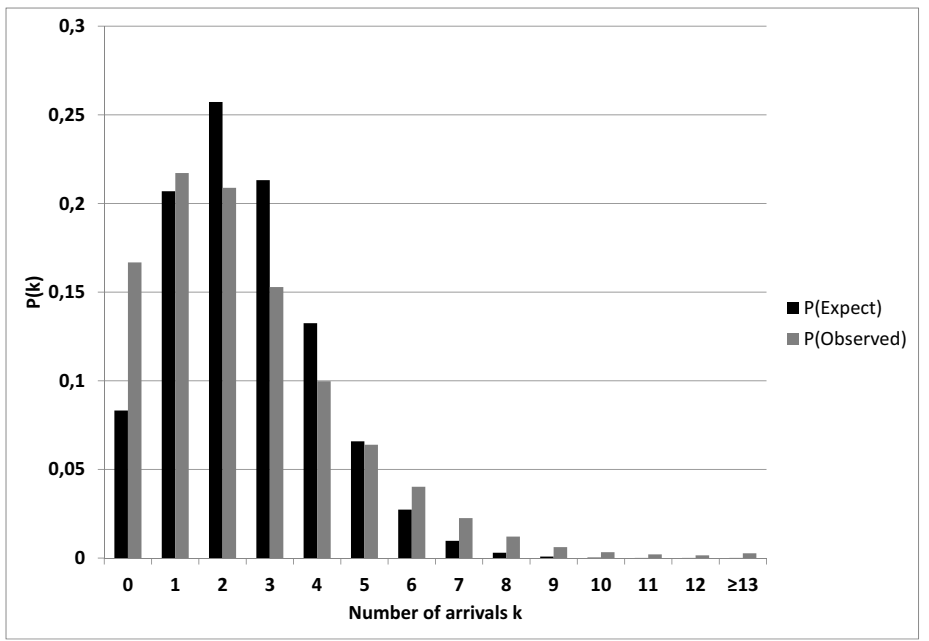

Fig. 4. Observed arrivals over New York City against Poisson hypothesis, $r=200 \mathrm{~km}$

Aircraft arrivals in the urban scenario over New York City are not following a Poisson distribution according to the Chi Square test. The empiric distribution and the Poisson distribution with the same mean value is shown in Figure 4. Both distributions are obviously different. The Chi Square test value exceeds the critical value by far, as shown in Table 1. As the hypothesis of arrivals and departures following a Poisson distribution must be rejected, a new one is formulated. Further suitable distributions are examined with focus on generalization of the Poisson distribution. The negative binomial distribution is a generalization of the Poisson distribution with a second parameter allowing to independently select the mean and variance. The Probability Density Function (PDF) of a negative binomial distribution is stated in Equation 3

$$
P(X=x)=\left\{\begin{array}{l}
p^{n}(1-p)^{x}\left(\begin{array}{c}
n+x-1 \\
n-1
\end{array}\right) x \geq 0 \\
0
\end{array}\right.
$$


Therefore, the hypothesis for the Chi Square test is changed and the data set is tested against the negative binomial distribution. As shown in [8], the negative binomial distribution is a mixture of Poisson distributions. Whereas for the Poisson distribution hypothesis only the mean value has to be estimated, for the negative binomial hypothesis an additional estimation of the variance is needed. Hence, the degree of freedom for the Chi Square test is increased by one. As visible in Figures 5, 6] and Table 2, the aircraft arrivals follow a negative binomial distribution.

Table 1. Overview: Chi Square test results against Poisson hypothesis

\begin{tabular}{|c|c|c|c|c|c|}
\hline Scenario & $\begin{array}{c}r_{\text {Spotbeam }} \\
{[\mathrm{km}]}\end{array}$ & $\begin{array}{c}\text { Mean arrivals } \\
\text { Interval }\end{array}$ & $\begin{array}{c}\text { Chi Square } \\
\text { test value }\end{array}$ & $\begin{array}{c}\text { Critical } \\
\text { value }\end{array}$ & $\begin{array}{c}\text { Test } \\
\text { result }\end{array}$ \\
\hline Intercontinental & $200 \mathrm{~km}$ & 0,1275 & 3,0836 & 3,84 & pass \\
\hline Rural & $200 \mathrm{~km}$ & 0,4052 & 367,82 & 7,82 & fail \\
\hline Urban & $200 \mathrm{~km}$ & 2,49 & 5960 & 19,68 & fail \\
\hline
\end{tabular}

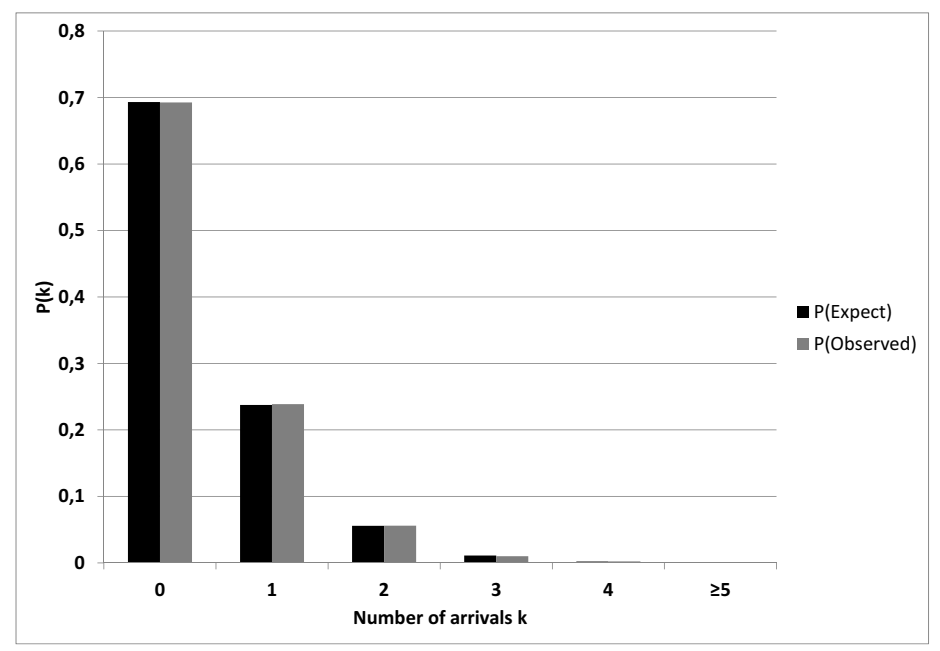

Fig. 5. Observed arrivals over Omaha against negative binomial hypothesis, $r=200 \mathrm{~km}$

The results show that inside the observed areas, the aircraft arrivals and departures follow a negative binomial distribution. It is therefore suitable to assume a negative binomial for aircraft arrivals and departures in those areas. As the three investigated areas cover representative flight scenarios, it can be assumed that this model can also be applied for air traffic in other areas. Even the first assumption of poissonian aircraft arrivals remains valid since the Poisson distribution is a special case of the negative binomial distribution. 


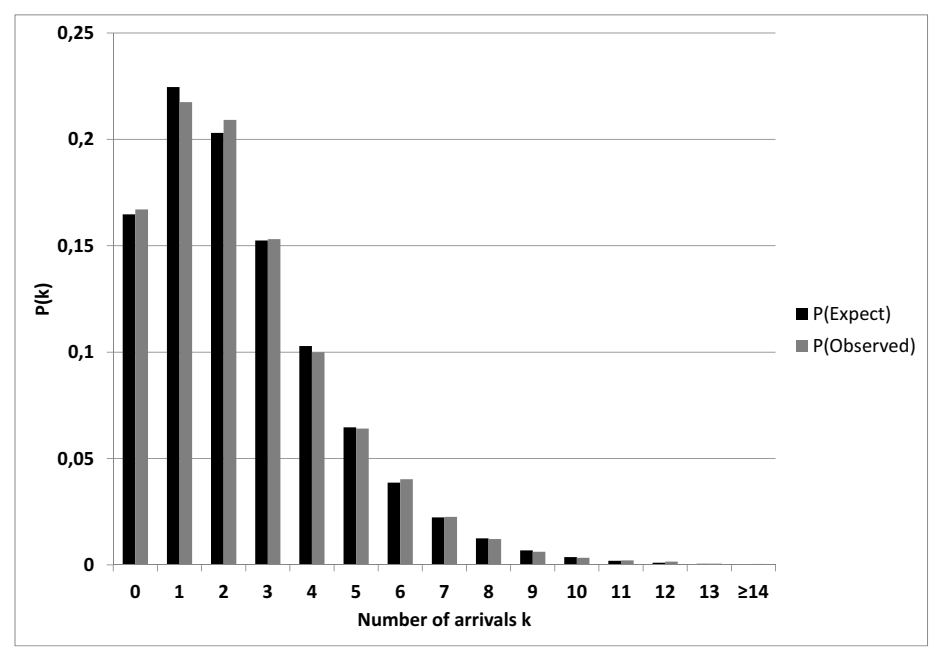

Fig. 6. Observed arrivals over New York City against negative binomial hypothesis, $r=200 \mathrm{~km}$

Table 2. Overview: Chi Square test results against negative binomial hypothesis

\begin{tabular}{|c|c|c|c|c|c|c|}
\hline Scenario & $\begin{array}{c}r_{\text {Spotbeam }} \\
{[\mathrm{km}]}\end{array}$ & $\begin{array}{c}\text { Mean arrivals } \\
\text { Interval }\end{array}$ & Variance & $\begin{array}{c}\text { Chi Square } \\
\text { test value }\end{array}$ & $\begin{array}{c}\text { Critical } \\
\text { value }\end{array}$ & $\begin{array}{c}\text { Test } \\
\text { result }\end{array}$ \\
\hline Intercontinental & $200 \mathrm{~km}$ & 0,1275 & 0.1339 & 0.3044 & 3,84 & pass \\
\hline Rural & $200 \mathrm{~km}$ & 0,4052 & 0.4494 & 7.5867 & 7,82 & pass \\
\hline Urban & $200 \mathrm{~km}$ & 2,49 & 4.4252 & 16,505 & 19,68 & pass \\
\hline
\end{tabular}

\section{Conclusion}

Aircraft position data has been collected and analyzed regarding aircraft arrivals and departures within time intervals of $\Delta t=10 \mathrm{~s}$. The investigations focussed on an intercontinental, rural, and urban area. In all areas, the stochastical aircraft arrival process is very similar to the departure process and follows a negative binomial distribution.

\section{Future Work}

In this work, we found out that the aircraft arrivals and departures inside a satellite spotbeam follow a negative binomial distribution. Future work should further clarify the reasons for this. Inside one spotbeam, especially in urban areas, arrival and departure rates of different aircraft types occur. While long range flights often just pass by, the impact of short haul flights e.g. by take off or landing is higher inside an urban spotbeam. This would also be an explanation for the varying results of the Chi Square test against the Poisson hypothesis. 
For futher validation of the hypothesis more regions have to be investigated. Thereby, generally valid distribution parameter (mean value and variance) can be determined.

\section{References}

1. Gramaglia, M., Serrano, P., Hernandez, J.A., Calderon, M., Bernardos, C.J.: New insights from the analysis of free flow vehicular traffic in highways. In: 2011 IEEE International Symposium on World of Wireless, Mobile and Multimedia Networks (WoWMoM), pp. 1-9 (2011), http://dx.doi.org/10.1109/WoWMoM.2011.5986384

2. Wisitpongphan, N., Bai, F., Mudalige, P., Sadekar, V., Tonguz, O.: Routing in sparse vehicular ad hoc wireless networks. IEEE Journal on Selected Areas in Communications 25(8), 1538-1556 (2007), http://dx.doi.org/10.1109/JSAC.2007.071005

3. Medina, D., Hoffmann, F., Rossetto, F., Rokitansky, C.-H.: A geographic routing strategy for north atlantic in-flight internet access via airborne mesh networking. IEEE/ACM Transactions on Networking (TON) 20(4), 1231-1244 (2012)

4. Medina, D., Hoffmann, F., Ayaz, S., Rokitansky, C.H.: Feasibility of an aeronautical mobile ad hoc network over the north atlantic corridor. In: 5th Annual IEEE Communications Society Conference on Sensor, Mesh and Ad Hoc Communications and Networks, SECON 2008, pp. 109-116 (2008), http://dx.doi.org/10.1109/SAHCN.2008.23

5. Flightradar24 real time flight tracking service, http://www.flightradar24.com

6. Fossa, C.E., Raines, R.A., Gunsch, G.H., Temple, M.A.: An overview of the IRIDIUM (r) low earth orbit (LEO) satellite system. In: Proceedings of the IEEE 1998 National Aerospace and Electronics Conference, NAECON 1998, pp. 152-159 (1998), http://dx.doi.org/10.1109/NAECON.1998.710110

7. Walke, B.: Mobilfunknetze und ihre Protokolle 2, pp. 433-493. Springer DE (2001)

8. Hofmann, M.: Uber zusammengesetzte poisson-prozesse und ihre anwendungen in der unfallversicherung. Ph.D. dissertation, Diss. Math. ETH Zurich, Nr. 2511, 0000. Ref.: Saxer, W., Korref.: Nolfi, P. (1955) 